\title{
MACC1 Suppresses Cell Apoptosis in Hepatocellular Carcinoma by Targeting the HGF/c-MET/AKT Pathway
}

\author{
Yingmin Yao Chanwei Dou Zhongtang Lu Xin Zheng Qingguang Liu \\ Department of Hepatobiliary Surgery, the First Affiliated Hospital of Xi'an Jiaotong University, Xi'an, \\ China
}

\section{Key Words}

MACC1 • Hepatocellular carcinoma • Apoptosis • HGF/c-MET signaling • AKT

\begin{abstract}
Background \& Aims: To investigate the expression and prognostic value of MACC1 in patients with HCC and identify the mechanism by which MACC1 inhibits HCC cell apoptosis. Methods: MACC1 and p-AKT expression was studied using immunohistochemistry of both HCC tissues and adjacent liver tissues. QRT-PCR and western immunoblotting were used to examine the expression of target genes at the mRNA and protein levels, respectively. The MTT assay was used to assess cell viability, and cell apoptosis was determined by DAPI staining, Annexin V/PI staining and Caspase 3/7 assay. Nude mice were used to perform in vivo experiments. Results: The overexpression of MACC1 was found in HCC tissues and was correlated with poor postsurgical prognosis. There was a positive relationship between MACC1 and p-AKT expression in HCC tissues. In vitro experiments showed that MACC1 repressed HCC cell apoptosis and promoted cell growth. Knockdown of c-MET abolished the anti-apoptotic function of MACC1. Next, MACC1 was verified to activate PI3K/AKT signaling by sensitizing HGF/C-MET signaling in HCC. MACC1 overexpression enhanced the HGF-driven phosphorylation of BAD, Caspase 9 and FKHRL1 and inhibited their pro-apoptotic functions in HCC cells. Finally, MACC1 was shown to inhibit cell apoptosis and promote HCC growth in vivo. Conclusions: This investigation revealed that MACC1 overexpression predicted worse prognosis after liver resection, which was attributed to the repression of HCC cell apoptosis via a molecular mechanism in which MACC1 accelerated the activation of the HGF/c-MET/PI3K/ AKT pathway and phosphorylated BAD, Caspase 9 and FKHRL1, ultimately preventing their nuclear translocation and their pro-apoptotic function.

Xin Zheng, MD., Ph.D.

or Qingguang Liu, MD., Ph.D.

KARGER 125
Department of Hepatobiliary Surgery, The First Affiliated Hospital of Xi'an Jiaotong University, 277 Yanta West Road, Xi an, 710061 (China)

E-Mail xin.zheng.zheng@gmail.com, E-Mail raymondzhengxin@foxmail.com 


\section{Introduction}

Hepatocellular carcinoma (HCC) has a mounting annual incidence of 4.9 per 100,000 persons, which makes it one of the most common cancers and the third leading cause of cancer-related deaths worldwide [1,2]. Despite recent advances in the diagnosis and treatment of HCC, the prognosis for HCC patients remains poor due to a low rate of curative resection and high incidence of postoperative tumor recurrence. Therefore, there is an urgent need to develop novel therapeutic strategies for HCC.

In 2009, Stein and her colleagues [3] identified by genome-wide search the metastasis associated with colon cancer 1 (MACC1) gene as the critical pro-metastatic factor in human colon cancer. MACC1 has been demonstrated to activate the HGF/c-MET pathway through binding to the promoter of c-MET. Several studies have revealed that aberrant activity of c-MET results in multiple cellular responses regulating proliferation, cell morphogenesis, migration and breakdown of the extracellular matrix [4-6]. In the event of activation by HGF, c-MET transmits intracellular signals and activates downstream Ras-mitogen-activated protein kinase (MAPK)/Erk and phosphoinositide 3-kinase (PI3K)/AKT pathways [7, 8], consequently promoting cell survival, migration and invasion and suppressing apoptosis in a variety of cancers.

PI3K is one of the important kinases regulating cell survival, proliferation and differentiation. Previous investigation demonstrated that PI3K assembles in the membrane by interacting with activated receptors directly or indirectly in response to stimulation by growth factors and cytokines. In consequence, the activated PI3K converts phosphatidylinositol(3-5)-trisphosphate (PIP3) from phosphatidylinositol 4,5-bisphosphate (PIP2) by phosphorylating inositols on the lipid membrane. PIP3 then binds with AKT, a type of serine-threonine kinase, and phosphorylates it directly. Phosphorylated AKT (p-AKT) phosphorylates its downstream signaling effectors such as Bad [9] and FKHRL1 [10] and in turn exerts an anti-apoptotic effect. However, to date there have been no studies determining whether MACC1 could inhibit cell apoptosis via the modulation of the HGF/c-MET/AKT pathway in HCC.

In our preliminary study, we found significantly more expression of MACC1 in HCC tissues compared with adjacent liver tissues, and the nuclear expression of MACC1 was correlated with poor tumor differentiation, intrahepatic metastasis and portal invasion. The ablation of MACC1 in HCC cells repressed cell migration and invasion by down-regulating MMP2, MMP9 and c-MET [11]. Here, we attempt to answer the following questions: 1. Does MACC1 mediate cell apoptosis of HCC cells? 2. Does MACC1 regulate the HGF/c-MET/ AKT pathway? 3. Is the phosphorylation of AKT attributed to MACC1-driven anti-apoptotic function in HCC cells? 4. Does MACC1 affect the growth of HCC xenografts?

\section{Materials and Methods}

\section{Reagents}

TurboFect transfection reagent and a RevertAid Premium First Strand cDNA Synthesis kit were purchased from Thermo (MA, USA). Full length cDNA of MACC1 was amplified from human embryonic kidney 293FT cells and cloned into the pCMV6 entry vector. The shRNA sequences targeting MACC1 were synthesized by GenePharma (Shanghai, China). The sequences were 5'-50-CAC CGA AGA TTG GAC TTG TAC ACT GCT TCA AGA GAG CAG TGT ACA AGT CCA ATC TTT TTT TTG-3' and 5'-GAT CCA AAA AAA AGAT TGG ACT TGT ACA CTG CTC TCT TGA AGC AGT GTA CAA GTC CAA TCT TC-3'. The negative shRNA sequences were 5'-CAC CGT TCT CCG AAC GTG TCA CGT CAA GAG ATT ACG TGA CAC GTT CGG AGA ATT TTT TG-3' and 5'GAT CCA AAA AAT TCT CCG AAC GTG TCA CGT AAT CTC TTG ACG TGA CAC GTT CGG AGA AC-3'. All shRNA sequences were recombined into a plasmid with a U6 promoter and green fluorescent protein, which was also obtained from GenePharma (Shanghai, China). The primary anti-MACC1 antibody (Cat. No.: ab106579) was obtained from Abcam (Cambridge, UK). The primary anti- p -c-MET antibody (Cat. No.: 3126), anti-AKT antibody (Cat. No.: 9272), anti- p-AKT antibody (Cat. No.: 3787), anti-Bad antibody (Cat. No.: 9239), anti- 
p-Bad antibody (Cat. No.: 5284), anti-FKHRL1 antibody (Cat. No.: 2497) and anti-p-FKHRL1 antibody (Cat. No.: 9466) were from Cell Signaling (MA, USA). The primary antibodies against $\beta$-actin (Cat. No.: sc-8432), anti-c-MET antibody (Cat. No.: sc-10), c-MET siRNA (Cat. No.: sc-29397) and relevant control siRNA (Cat. No.: sc-37007) were purchased from Santa Cruz Biotechnology (CA, USA). The PI3K inhibitor LY294002 was purchased from Beyotime Institute of Biotechnology (Haimen, China). The Annexin V-FITC Apoptosis Detection kit was from BD Biosciences (Cat. No.:556547). The Apo-ONE® Homogeneous Caspase-3/7 Assay kit (Cat. No.:G7792) was from Promega (WI, USA). The Fastgen200 RNA isolation kit was obtained from Fastgen Co. (Shanghai, China). SYBR Green PCR master mix was purchased from TaKaRa Biotechnology (Dalian, China). The BCA Protein Assay kit was from Beyotime Co. (Haimen, China). The HyGLO HRP detection kit was from Denville (Metuchen, NJ, USA). The Alexa Fluor ${ }^{8} 488$ Annexin V/Dead Cell Apoptosis kit and Lipofectamine RNAi MAX Reagent were from Invitrogen (CA, USA).

\section{Cell lines and culture}

The human HCC cell line MHCC97H was provided by Prof. Kefeng Dou from the Fourth Military Medical University (Xi'an, China) and was cultured with DMEM medium. SMMC7721 cells and Hep3B cells were both obtained from the American Type Culture Collection and grown in RPMI-1640 medium and complete MEM medium, respectively. The cell culture medium was supplemented with $10 \%$ heat-inactivated fetal calf serum (FBS).

\section{Establishment of MACC1 stable transfectant clones}

Both MACC1-expressing plasmid and control plasmid were transfected into Hep3B cells and SMMC7721 cells using TurboFect transfection reagent. The MACC1 stable transfectant clones and control clones were obtained after 2-week selection with Geneticin (G418) from Invitrogen (Carlsbad, CA, USA) (the concentration of G418 was $500 \mathrm{~g} / \mathrm{mL}$ for Hep3B cells and $300 \mathrm{~g} / \mathrm{mL}$ for SMMC7721 cells).

\section{ShRNAs transfection}

The plasmids with shRNA against MACC1 or scrambled shRNA were transfected into MHCC97 cells with the help of TurboFect transfection reagent. Cells were observed $24 \mathrm{~h}$ after transfection with a fluorescence microscope to select clones with more than an $80 \%$ transfection rate for future experiments of this study.

\section{SiRNAs transfection}

HCC cells were seeded in six-well plates at the concentration of $0.2 \times 10^{6}$ per well and grown overnight. A total of $100 \mathrm{nM}$ siRNA was transfected into HCC cells in each well using Lipofectamine RNAi MAX Reagent in accordance with the manufacturer's instructions. The cells were used for further experiments $48 \mathrm{~h}$ after transfection.

Quantitative real-time reverse transcription polymerase chain reaction (qRT-PCR)

Total RNA was extracted from HCC cells with the Fastgen200 RNA isolation kit. Then, it was reverse transcribed into cDNA using a RevertAid Premium First Strand cDNA Synthesis kit according to the manufacturer's protocol. SYBR Green PCR master mix was used to perform qRT-PCR with an IQ5 System. The primers to detect the mRNA level of MACC1 were 5'-CTTGCGGAGGTCACCATAGC-3' and 5 '-GATTTCCAACAACGGGCTCA-3'. $\beta$-actin was used as the internal control. The primers to detect $\beta$-actin mRNA were 5'-GGGAAATCGTGCGTGACAT-3' and 5'-CTGGAAGGTGGACAGCGAG-3'. MACC1-expressing plasmid was used to create the standard curve. All samples were normalized to the internal control. In total, we performed this measurement with 3 pairs of samples, and there were three replicates for each examination.

\section{Western immunoblotting}

The protocol for western immunoblotting has been described in our previous paper [12]. Briefly, cell lysates were prepared in RIPA buffer with inhibitors of proteases and with sodium orthovanadate as an inhibitor of phosphotases. The protein concentration was examined using the BCA Protein Assay kit and spectrophotometry. Denatured protein $(30 \mu \mathrm{g})$ was separated by electrophoresis in SDS-PAGE gels and transferred onto PVDF membranes. The protein blots in PVDF membranes were incubated with primary antibody overnight at $4^{\circ} \mathrm{C}$. After 3 washes with TBST, blots were probed with secondary antibodies 
Yao et al.: MACC1 Represses HCC Cell Apoptosis

conjugated with HRP. Finally, HRP was detected using the HyGLO HRP detection kit. $\beta$-actin was measured as the internal control for equal loading.

\section{Cell viability assay}

HCC cells were seeded in 96-well plates at 5000 cells per well and incubated for 24 hours. The 3-(4, 5-dimethylthiazol-2-yl)-2,5-diphenyl tetrazolium bromide (MTT) assay was used to assess cell viability at $24,48,72$ and 96 hours. In total, we performed this measurement with 3 pairs of samples, and six replicates were set for each assessment.

\section{Cell apoptosis assessment}

We performed three distinct assessments to detect cell apoptosis in this study. Apoptotic nuclear changes including chromatin condensation and nuclear fragmentation were measured by staining cells with DAPI and visualizing them with fluorescence microscopy. The Apo-ONE Homogeneous Caspase-3/7 Assay kit was used to examine the caspase $3 / 7$ activity of HCC cells. The results were read using a Luminoskan. Populations of apoptotic cells were measured by staining HCC cells with Annexin V-FITC and PI labeling using the Alexa Fluor ${ }^{\circledR} 488$ Annexin V/Dead Cell Apoptosis kit in accordance with the manufacturer's recommendations. In total, we performed this detection with 3 pairs of samples and set 3 replicates for each assessment.

\section{In vivo experiments}

Two million MACC1-expressing or control Hep3B cells were mixed in $150 \mu \mathrm{L}$ of Matrigel and consequently injected subcutaneously into the flanks of 4- to 6-week-old male nude mice. Tumor size was measured with calipers every 7 days. All mice were sacrificed 3 weeks after the injection of HCC cells. Formalin-fixed, paraffin-embedded tumor samples were collected for the terminal deoxynucleotidyl transferase-mediated dUTP-biotin nick end labeling (TUNEL) assay. The TUNEL assay was used to evaluate cell apoptosis in the xenograft tissues as described previously [13]. All in vivo experiment protocols were approved by the Institutional Animal Care and Use Committee of the First Affiliated Hospital of Xi'an Jiaotong University.

\section{Immunohistochemistry staining}

A total of 50 HCC patients undergoing liver resection, including curative resection for early HCC and palliative resection for advanced HCC, from 2009 to 2010 in our hospital were recruited for this study. None of the patients received pre-operative chemotherapy or embolization. We obtained HCC tissues and adjacent liver tissues ( $>2 \mathrm{~cm}$ distance to the resection margin) that were immediately placed in paraformaldehyde upon removal during the operation. Clinical information, including Edmondson classification, tumor-nodemetastasis (TNM) staging, maximum tumor diameter and normal tumor-adjacent tissues, was confirmed by an experienced pathologist who was blinded to the clinical information.

Sections of $4 \mu \mathrm{m}$ thickness cut from formalin-fixed, paraffin-embedded blocks were mounted on glass slides. After deparaffinization, antigen retrieval was conducted by heating the slides below the boiling point for $20 \mathrm{~min}$. Endogenous peroxidase activity was blocked by incubation with $3 \%$ hydrogen peroxide in methanol for $10 \mathrm{~min}$. After being washed twice in phosphate-buffered saline (PBS) for $5 \mathrm{~min}$ each wash, sections were blocked by incubating with $3 \%$ bovine serum albumin (BSA) for 10 min. Primary anti-MACC1 antibody (dilation 1:100) and primary anti- p-AKT antibody (dilation 1:50) were both incubated at $4^{\circ} \mathrm{C}$ overnight. After being washed with PBS three times, sections were incubated with biotinylated secondary antibodies from Zhongshan Goldenbridge Biotechnology Ltd. Co. (Beijing, China) at $4{ }^{\circ} \mathrm{C}$ overnight. Sections were stained using the avidin-biotin-peroxidase complex (SABC) method, visualized with diaminobenzidine and counterstained with hematoxylin. All sections were dehydrated in alcohol prior to mounting.

Two experienced pathologists independently observed the sections. Semi-quantitative scoring methods included evaluating the staining intensity and the percentage of specifically positively stained HCC cells. The intensity of staining was divided into four grades: 0 , none; 1 , weak; 2 , moderate and 3 , strong. The percentage of specifically positively stained tumor cells was also classified into four grades: $0(<5 \%), 1(6 \%$ 25\%), 2 (26\%-50\%), 3 (51\%-75\%), and 4 (>75\%). The IHC staining score was calculated by multiplying the staining intensity and the percentage of specifically positively stained HCC cells. Ten independent high magnification fields $(\times 400)$ were assessed in each section, and the mean staining score was obtained. 


\section{Statistical analysis}

All data were expressed as the means and standard errors of the mean. The Mann-Whitney test or Student's t test was used to evaluate statistical significance of differences between groups. The correlation between MACC1 expression in HCC tissues, clinical characteristics and p-AKT expression in HCC tissues was analyzed using the Spearman rank test. Differences in the Kaplan-Meier survival curves between the high MACC1 group and low MACC1 group were assessed using the log rank test. A $P$ value less than 0.05 was considered statistically significant.

\section{Results}

MACC1 expression in HCC tissues was aberrantly increased and associated with $p$-AKT

To determine the expression of MACC1 in HCC tissues and its correlation with p-AKT, we performed an IHC staining assay of 50 pairs of HCC specimens and adjacent liver specimens. As shown in Fig. 1A, MACC1 protein was located mainly in the cytoplasm. Of the 50 cases, there were 37 HCCs (74\%) with higher MACC1 expression in HCC tissues than in adjacent liver tissues. After analyzing IHC staining scores, we found that the expression of MACC1 was significantly increased in HCC tissues ( $\mathrm{P}<0.001$, Fig. 1B). After Spearman rank analysis, we found that MACC1 expression in HCC tissues was clearly related to high EdmondsonSteiner classification $(\mathrm{r}=0.523, \mathrm{P}=0.020)$ and advanced TNM staging $(\mathrm{r}=0.249, \mathrm{P}=0.033)$, whereas no correlation was found between MACC1 expression in HCC tissues and gender $(\mathrm{r}=0.123, \mathrm{P}=0.220)$, age $(\mathrm{r}=0.356, \mathrm{P}=0.081)$, HBV infection $(\mathrm{r}=0.523, \mathrm{P}=0.161)$, liver cirrhosis ( $r=0.411, P=0.065)$, serum $\alpha$-fetoprotein (AFP) level $(r=0.221, P=0.082)$, or tumor size $(\mathrm{r}=0.271, \mathrm{P}=0.091)$.

To establish the role of MACC1 on prognosis of HCCs after liver resection, we performed a follow-up study on all 50 HCCs and obtained detailed information for 41 cases (82\%). The median duration of follow-up was 27 months. We observed the results of MACC1 staining in 5 pairs of HCC and adjacent liver tissue slides for each of the 41 HCCs and calculated the staining scores. The 41 HCCs were divided into two groups, the high MACC1 group and low MACC1 group, using the mean ratio of tumor/benign MACC1 expression as the cut-off value. The high MACC1 group $(n=28)$ included cases with higher MACC1 expression in HCC tissues than in adjacent liver tissues, whereas the low MACC1 group ( $n=13$ ) included patients expressing lower levels of MACC1 protein in HCC tissues. The 3-year postsurgical survival rate was dramatically lower in the high MACC1 group (9.9\%) than in the low MACC1 group (61.5\%). The comparison of the Kaplan-Meier curves of the overall survival rate after liver resection also showed that patients in the high MACC1 group had significantly shorter postsurgical survival time than those in the low MACC1 group ( $\mathrm{HR}=2.368 ; 95 \% \mathrm{Cl}$ : 1.062 , 5.708; P = 0.036; Fig. 1C).

We also detected p-AKT expression in these specimens to determine whether there is positive correlation between MACC1 and p-AKT expression in HCC tissues. Analysis showed that p-AKT protein was expressed mainly in both the cytoplasm and cell nucleus (Fig. 1A) and that the expression of p-AKT in HCC tissues was increased compared with expression in adjacent liver tissues (Fig. 2A). Spearman rank analysis confirmed that p-AKT expression was positively associated with MACC1 expression in HCC tissues (Fig. 2B). Similarly, p-AKT staining was examined in 5 pairs of HCC and adjacent liver tissue slides for each one of the 41 HCC patients. The mean ratio of tumor/benign p-AKT expression was calculated. We classified the 41 HCCs with follow-up information into two groups, including the high p-AKT group $(n=31)$ and low p-AKT group $(n=10)$, using the ratio of tumor/benign p-AKT expression as the cut-off value. The 3 -year survival rate after liver resection was $15.2 \%$ in the high p-AKT group, which was significantly lower than that (70\%) in the low p-AKT group. After analyzing the Kaplan-Meier curves, we found a tendency toward shorter overall survival after surgery in patients from the high p-AKT group compared with those in the low p-AKT group (HR = 2.715; 95\%Cl: 1.120, 6.581; P = 0.027; Fig. 1D). 
Fig. 1. A There was significantly higher MACC1 expression in HCC tissues (a) than in adjacent liver tissues (b). As indicated by the black arrow, most of the MACC1 protein was expressed in the cytoplasm. Similarly, p-AKT expression was increased in HCC tissues (c) compared with adjacent liver tissues (d). P-AKT protein staining was found in both the cell nucleus (white arrow) and cytoplasm (black arrow). Pictures of negative IHC staining of both HCC tissue (e) and adjacent liver tissue (f) are shown here as a control. B Analysis of IHC scores showed that MACC1 expression in HCC tissues was notably higher than expression in adjacent liver tissues. $\mathrm{C} \mathrm{Pa-}$ tients with lower MACC1 expression in HCC tissues had a better prognosis after liver resection than those with higher MACC1 expression in HCC tissues.
A
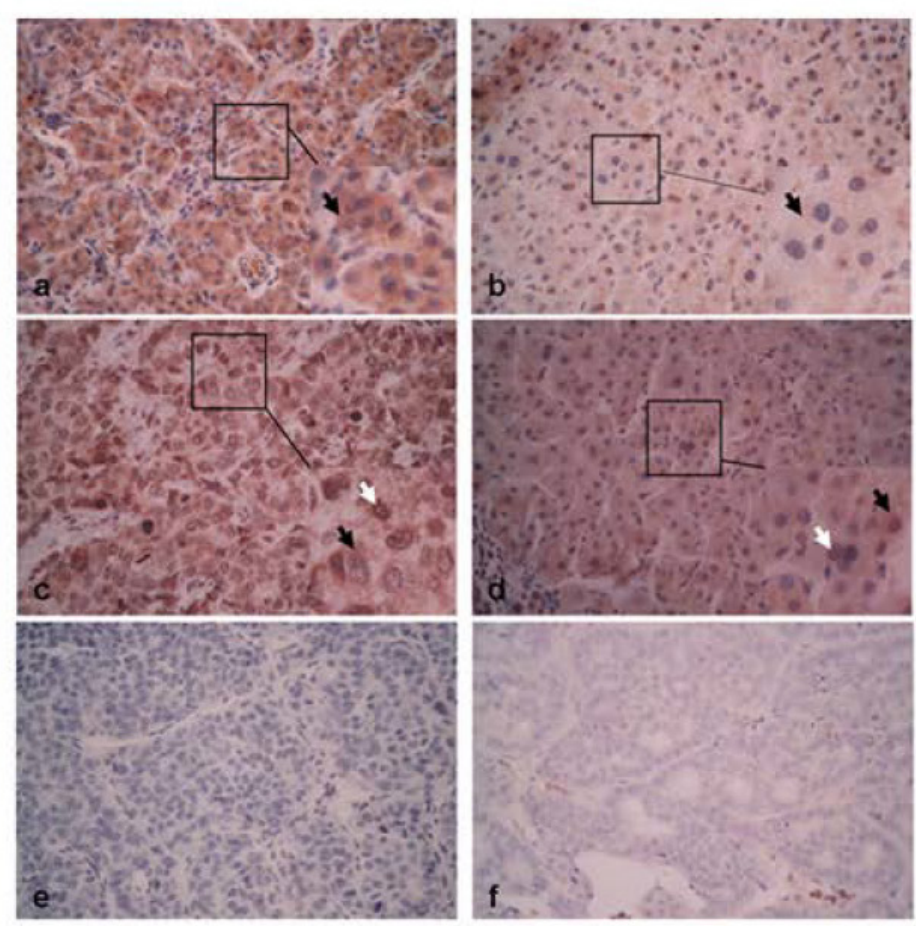

B
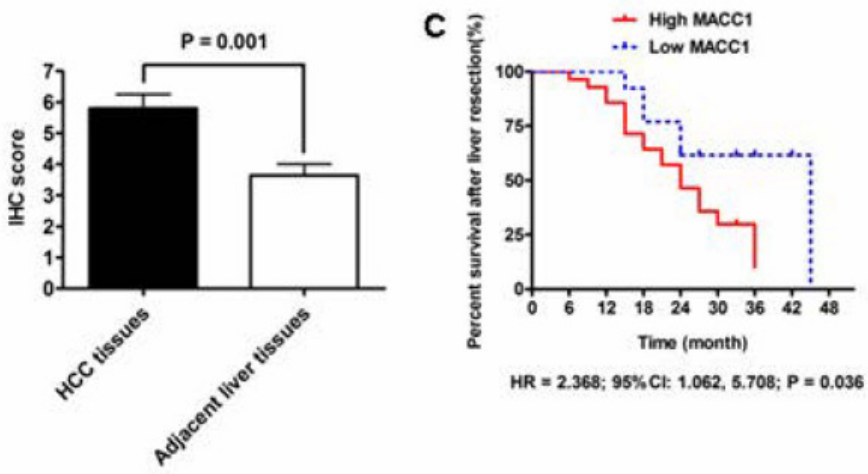

$H R=2.368 ; 95 \% C l: 1.062,5.708 ; P=0.036$

D

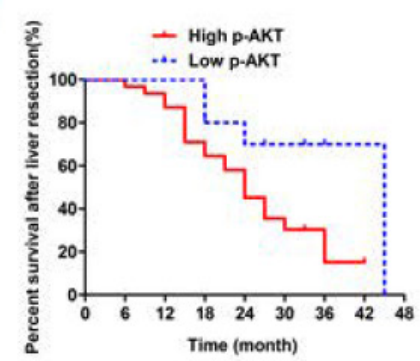

$H R=2.715 ; 95 \% \mathrm{Cl}: 1.120,6.581 ; P=0.027$

MACC1 repressed cell apoptosis and promoted cell growth in HCC cells

As shown in Fig. 3A, both qRT-PCR and western immunoblotting confirmed MACC1 overexpression in Hep3B cells after stable transfection of MACC1-expressing plasmid. A DAPI staining assay for apoptosis was performed to determine the effect of MACC1 on cell apoptosis. Assay results showed that the percentage of apoptotic cells in Hep3B cells with high levels of MACC1 (MACC1 Hep3B cells) was significantly lower than that in Hep3B cells with low levels of MACC1 (control Hep3B cells) (P < 0.001, Fig. 3B). More Caspase 3/7 
Fig. 2. A Mann-Whitney U testing showed that there was more p-AKT expression in HCC tissues than in adjacent liver tissues. B Spearman rank analysis revealed that MACC1 expression was positively associated with p-AKT expression in adjacent liver tissues.

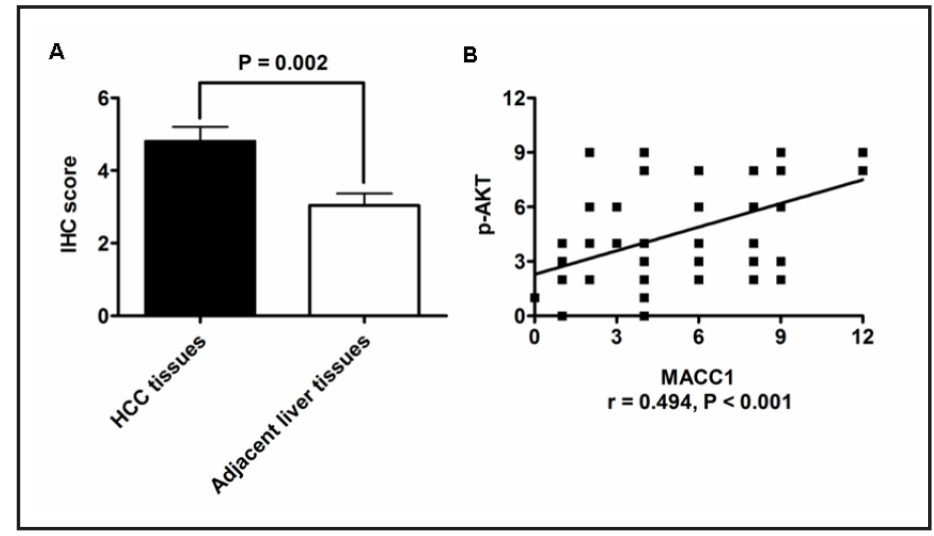

Fig. 3. A Transfection with MACC1-expressing plasmid up-regulated MACC1 expression in Hep3B cells at both the mRNA and protein levels. B DAPI staining assay showed that forced expression of MACC1 clearly decreased the percentage of apoptotic Hep3B cells. C There was lower activity of Caspase 3/7 in Hep3B cells after overexpression of MACC1. D The Flow cytometry assay showed that overexpression of MACC1 resulted in repression of cell apoptosis in Hep3B cells. E As assessed by MTT assay, MACC1 overexpression was found to enhance growth of Hep3B at 48 $\mathrm{h}, 72 \mathrm{~h}$ and $96 \mathrm{~h}$.

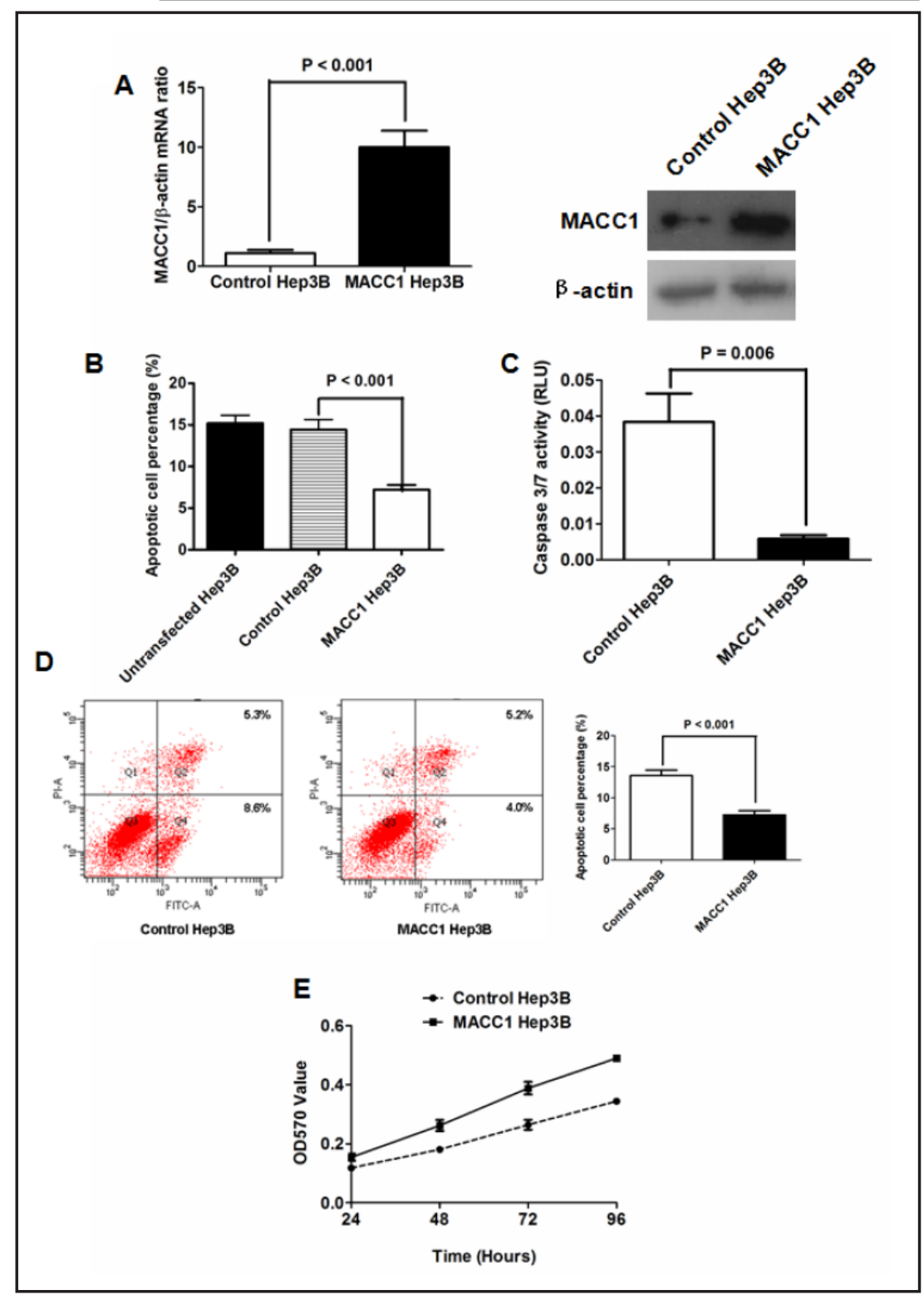

activity was found in control Hep3B cells than in MACC1 Hep3B cells (Fig. 3C). In addition, a flow cytometry assay showed that the percentage of early apoptotic cells and late apoptotic cells in the MACC1 Hep3B cell population was clearly lower than that in control Hep3B cells 
Fig. 4. A Both qRT-PCR and western immunoblotting showed that MACC1 SMMC7721 cells expressed more MACC1 than control SMMC7721. B Forced expression of MACC1 reduced the percentage of apoptotic SMMC7721 cells as shown by DAPI staining. C MACC1 overexpression also led to down-regulation of Caspase $3 / 7$ activity in SMMC7721 cells. D Forced expression of MACC1 accelerated growth of SMMC7721 cells.

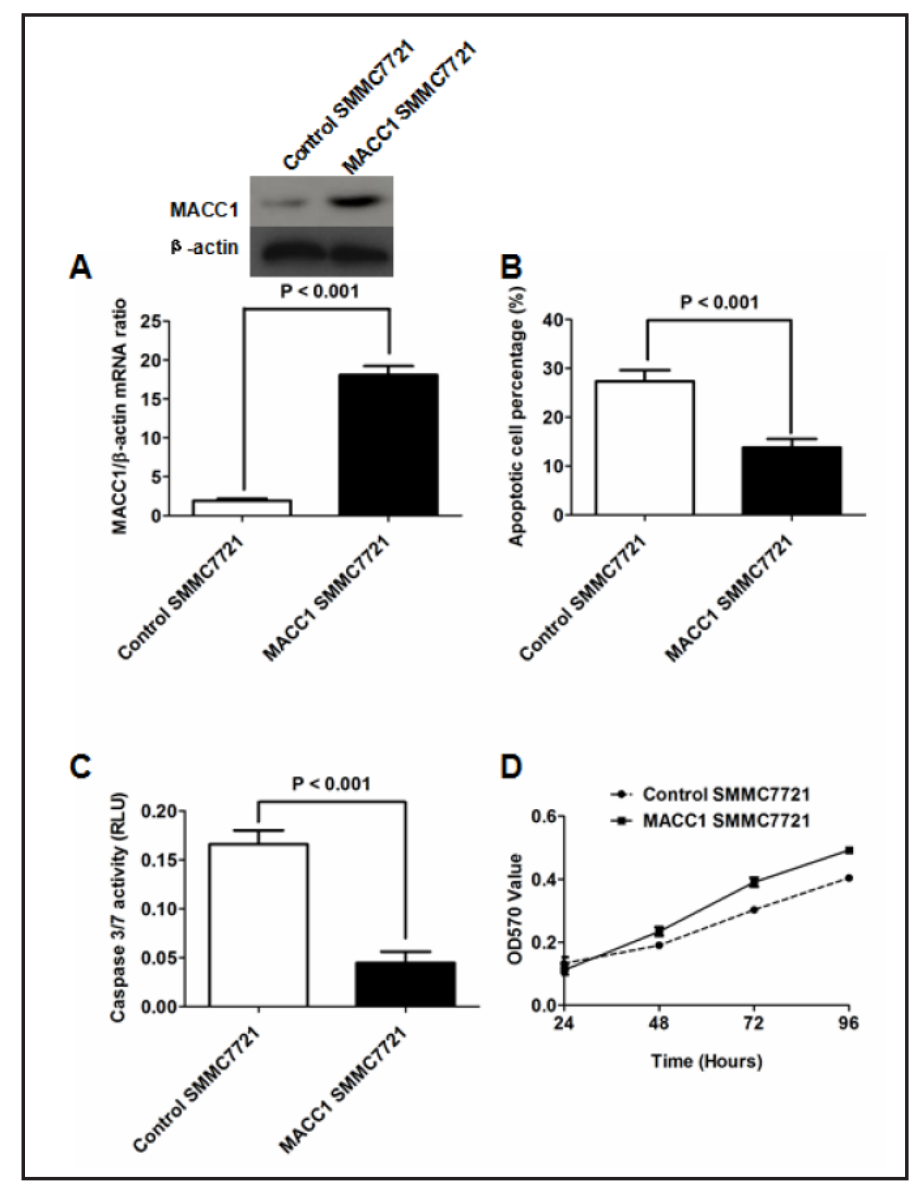

(Fig. 3D). Consequently, forced the expression of MACC1 increased Hep3B cell viability (as assessed by MTT assay) at all four time points evaluated (Fig. 3E).

We also examined the anti-apoptotic function of MACC1 in another HCC cell line, SMMC7721. As shown in Fig. 4A, MACC1 expression was increased greatly in SMMC7721 cells by transfection with a MACC1-expressing plasmid. As assessed by DAPI staining assay, there was a lower percentage of apoptotic SMMC7721 cells transfected with MACC1expressing plasmid (MACC1 SMMC7721 cells) compared with those transfected with control plasmid (control SMMC7721 cells) (Fig. 4B). Caspase 3/7 activity of SMMC7721 cells was significantly decreased by the overexpression of MACC1 (Fig. 4C). Consistently, MACC1 overexpression accelerated growth of SMMC7721 cells at certain time points from 48 to 96 hours, as shown in Fig. 4D.

Knockdown of MACC1 was performed in MHCC97 cells with high levels of MACC1 [11] to confirm its regulatory effect on cell apoptosis and growth. As shown in Fig. 5A, shRNA sequences targeting MACC1 successfully suppressed MACC1 expression in MHCC97 cells. The number of apoptotic nuclei was markedly increased by knockdown of MACC1 (Fig. 5B). MHCC97 cells with MACC1 shRNAs (MACC1 shRNA MHCC97) exhibited an over 2-fold decrease in caspase 3/7 activity compared with MHCC97 cells with scrambled shRNAs (Scr shRNA MHCC97) (Fig. 5C). The MTT assay showed that silencing MACC1 causes a remarkable inhibition of cell viability in MHCC97 cells (Fig. 5D). These experiments implied that MACC1 resulted in HCC cell apoptosis and growth arrest in vitro.

MACC1 exerted control over PI3K/AKT signaling by facilitating the activation of $H G F / c$ MET signaling in HCC

To explore the underlying mechanism whereby MACC1 impairs HCC cell apoptosis and growth, we examined whether MACC1 accelerated HGF/c-MET signaling activation. As 
Fig. 5. A Transfection with MACC1 shRNAs decreased MACC1 expression in MHCC97 as shown by both qRT-PCR and western immunoblotting. B Knockdown of MACC1 increased the percentage of apoptotic MHCC97 cells as assessed by DAPI staining assay. C Silencing of MACC1 significantly enhanced Caspase 3/7 activity in MHCC97 cells. D MTT assay verified that growth of MHCC97 cells was suppressed by silencing MACC1.

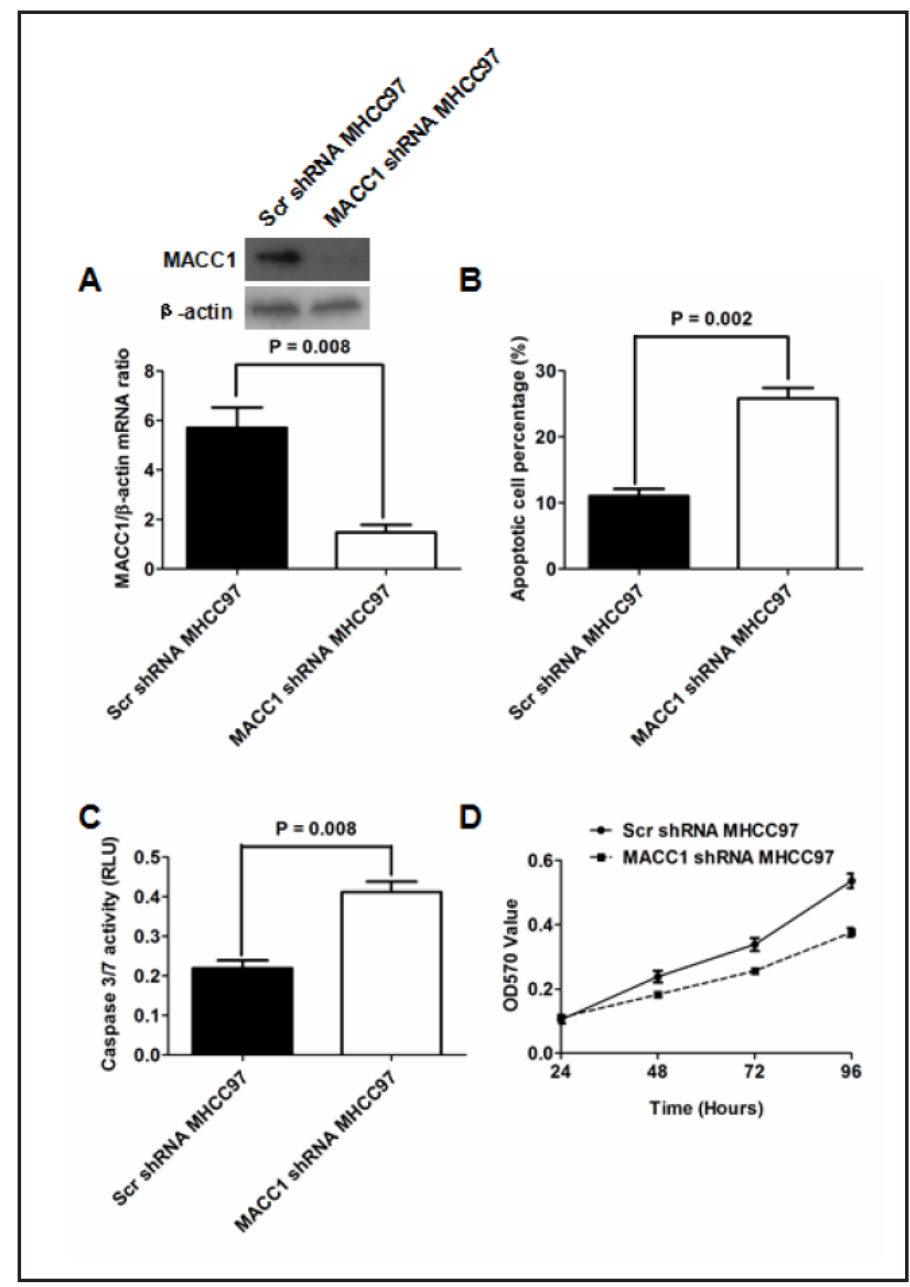

shown in Fig. 6A, forced expression of MACC1 led to the up-regulation of c-MET in Hep3B cells. After HGF treatment overnight at a concentration of $20 \mathrm{ng} / \mathrm{mL}$, we observed that there was more expression of both c-MET and p-c-MET in MACC1 Hep3B cells than in control Hep3B cells (Fig. 6A). Consequently, the overexpression of MACC1 strongly increased the phosphorylation of AKT in response to HGF (Fig. 6B). Specific PI3K inhibitor LY294002 treatment abolished MACC1-induced phosphorylation of AKT in Hep3B cells.

MACC1 overexpression enhanced the HGF-driven phosphorylation of apoptosis-related proteins BAD, Caspase 9 and FKHRL1 and inhibited their pro-apoptotic function in HCC cells

Bcl-2-associated death promoter (BAD), Caspase 9 and FKHRL1 (FOXO3) are renowned pro-apoptotic proteins that have been considered downstream effectors of PI3K/AKT signaling. As assessed by western immunoblotting, the phosphorylation of BAD, Caspase 9 and FKHRL1 induced by HGF treatment was enhanced after MACC1 overexpression (Fig. 6C). These data suggest that MACC1 mediated AKT phosphorylation via the HGF/c-MET/ PI3K pathway, increased the phosphorylation of BAD, Caspase 9 and FKHRL1, prevented their nuclear translocation and consequently inhibited HCC cell apoptosis.

C-MET played a critical role in the anti-apoptotic function of MACC1 in HCC cells

To shed more light on the role of c-MET in cell apoptosis induced by MACC1, we repressed c-MET expression in Hep3B by transfecting with targeting siRNAs before forced expression of MACC1. that the up-regulation of c-MET induced by MACC1 was abated as shown by both qRT-PCR and western immunoblotting (Fig. 7A). The percentage of cells 


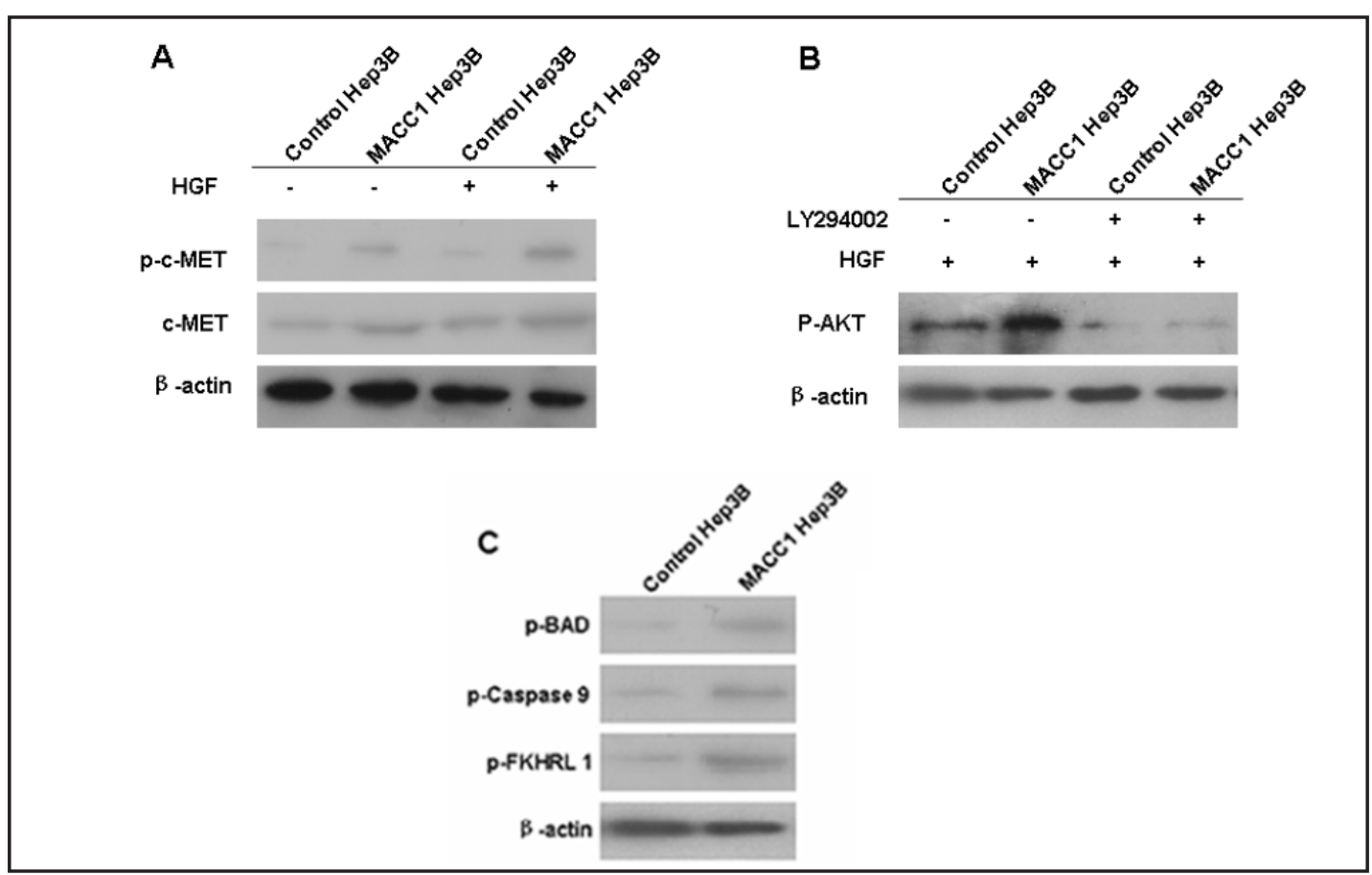

Fig. 6. A MACC1 overexpression was found to not only increase c-MET protein but also promote the HGF-driven up-regulation of c-MET phosphorylation in Hep3B cells. B Forced expression of MACC1 dramatically accelerated phosphorylation of AKT protein in Hep3B cells treated with HGF. Moreover, the PI3K-specific inhibitor LY294002 abolished AKT phosphorylation induced by HGF in both control and MACC1 Hep3B cells, which indicates that MACC1 plays an important part in HGF-induced activation of PI3K/AKT signaling and in turn mediates HCC cell apoptosis. C In Hep3B cells, forced expression of MACC1 significantly increased phosphorylation of BAD, Caspase 9 and FKHRL1.

with apoptotic nuclei, as indicated by DAPI staining, did not increase in MACC1 Hep3B cells compared with control Hep3B cells (Fig. 7B). Similarly, no difference in caspase 3/7 activity was found between MACC1 Hep3B cells and control Hep3B cells after silencing c-MET (Fig. 7C). Thus, the anti-apoptotic effect of MACC1 on HCC cells was attributed to c-MET.

MACC1 overexpression promoted tumorigenicity of HCC cells in nude mice

To test the effect of MACC1 on HCC growth in vivo, we subcutaneously inoculated nude mice with MACC1 Hep3B cells or control Hep3B cells in the right flank regions. Tumorigenicity was significantly higher in MACC1 Hep3B cells. As shown in Fig. 8A, remarkably rapid tumor growth was observed in the MACC1 Hep3B cell group after three weeks. Moreover, the TUNEL assay showed that there were notably fewer apoptotic cells in the xenograft tissues of MACC1 Hep3B cells than those of control Hep3B cells (Fig. 8B). The results suggested that MACC1 accelerated HCC growth in nude mice by repressing cell apoptosis.

\section{Discussion}

HCC is one of the few malignant tumors in which a continued increase in incidence has been observed worldwide for several years. Every year, there are approximately 750,000 new HCC cases reported worldwide $[2,14]$. Recently, the literature reported that the incidence rate of HCC approximated its death rate, which indicates that most HCC patients ultimately die of this disease [2]. Accordingly, there has been a continuous focus on HCC treatment. To date, liver resection at an early stage of HCC remains the gold standard for patients with normal liver function. Unfortunately, due to the high rate of recurrence or de novo tumor emergence 


\begin{tabular}{|c|c|c|}
\hline Cellular Physiology & Cell Physiol Biochem 2015;35:983-996 & \\
\hline and Biochemistry & $\begin{array}{l}\text { DOI: 10.1159/000369754 } \\
\text { Published online: rebruary 02, } 2015\end{array}$ & $\begin{array}{l}\text { O } 2015 \text { S. Karger AG, Basel } \\
\text { www.karger.com/cpb }\end{array}$ \\
\hline
\end{tabular}

Fig. 7. A C-MET siRNAs successfully silenced c-MET expression in both control and MACC1 Hep3B cells. B DAPI staining assay showed that knockdown of c-MET abolished the anti-apoptotic function of MACC1 in Hep3B cells. C Forced expression of MACC1 did not affect Caspase 3/7 activity in Hep3B.
Fig. 8. A The size of MACC1 Hep3B cell xenografts was significantly larger than control Hep3B cell xenografts during the 21 days after HCC cells were inoculated into nude mice. B TUNEL assay showed that there were fewer apoptotic cells in xenograft tissues from MACC1 Hep3B cells (a) than in xenograft tissues from control Hep3B cells (b).

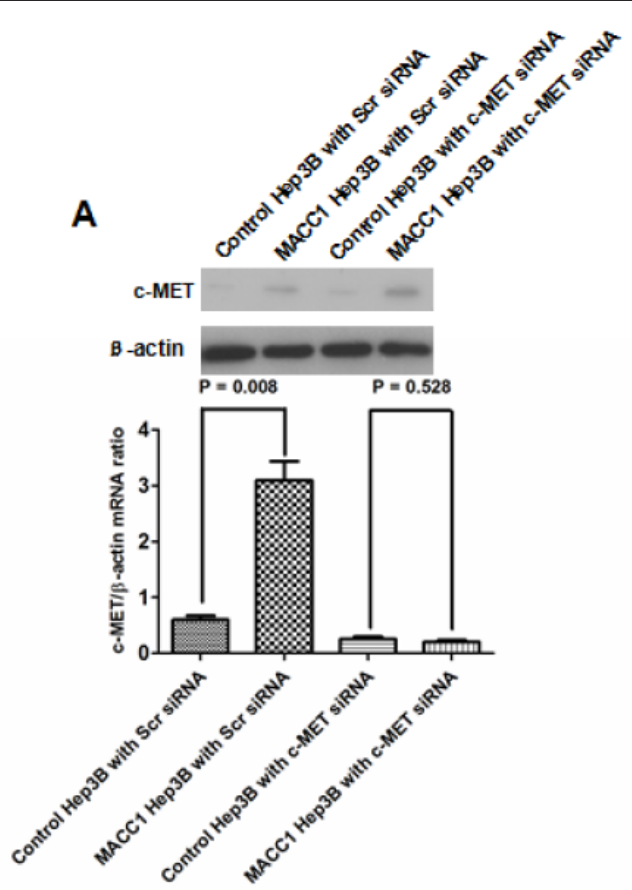

B

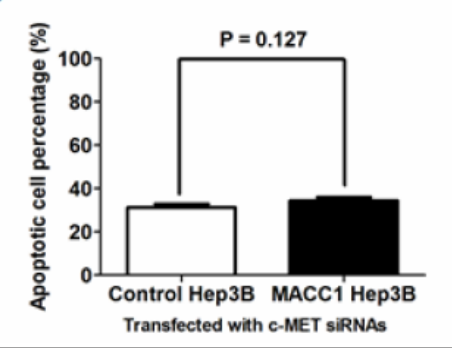

C

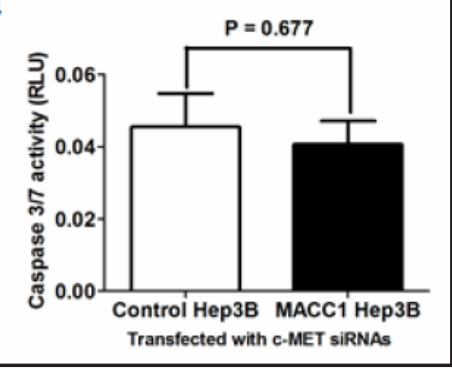

A

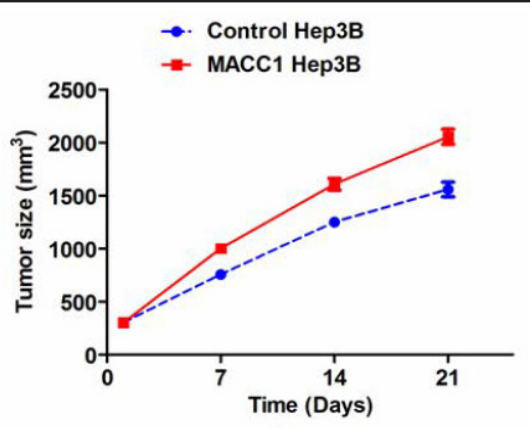

B
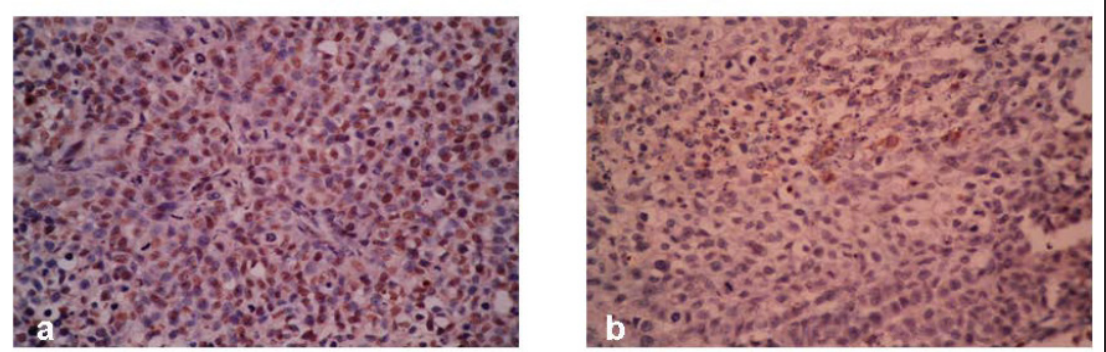

in the liver remnant, the recurrence rate after curative liver resection is approximately 50\% at 2 years and $75 \%$ at 5 years as reported by a growing number of clinical studies [15]. KARGER 
Fig. 9. Working model of the molecular mechanism whereby MACC1 mediates cell apoptosis in HCC cells. A When MACC1 is expressed at low levels, BAD, Caspase 9 and FKHRL1 in the cytoplasm enter into the nucleus, regulate the transcription of their target genes, and consequently induce cell apoptosis. B When MACC1 is overexpressed, MACC1 increases c-MET expression by binding with the promoter of c-MET; thus, HGF/cMET signaling is activated. Activated HGF/ c-MET signaling promotes phosphorylation of BAD, Caspase 9 and FKHRL1 via PI3K/AKT signaling. P-BAD, p-Caspase 9 and p-FKHRL1 cannot translocate into the cell nucleus and exert their pro-apoptotic effect. Ultimately, cell apoptosis is inhibited in HCC cells.

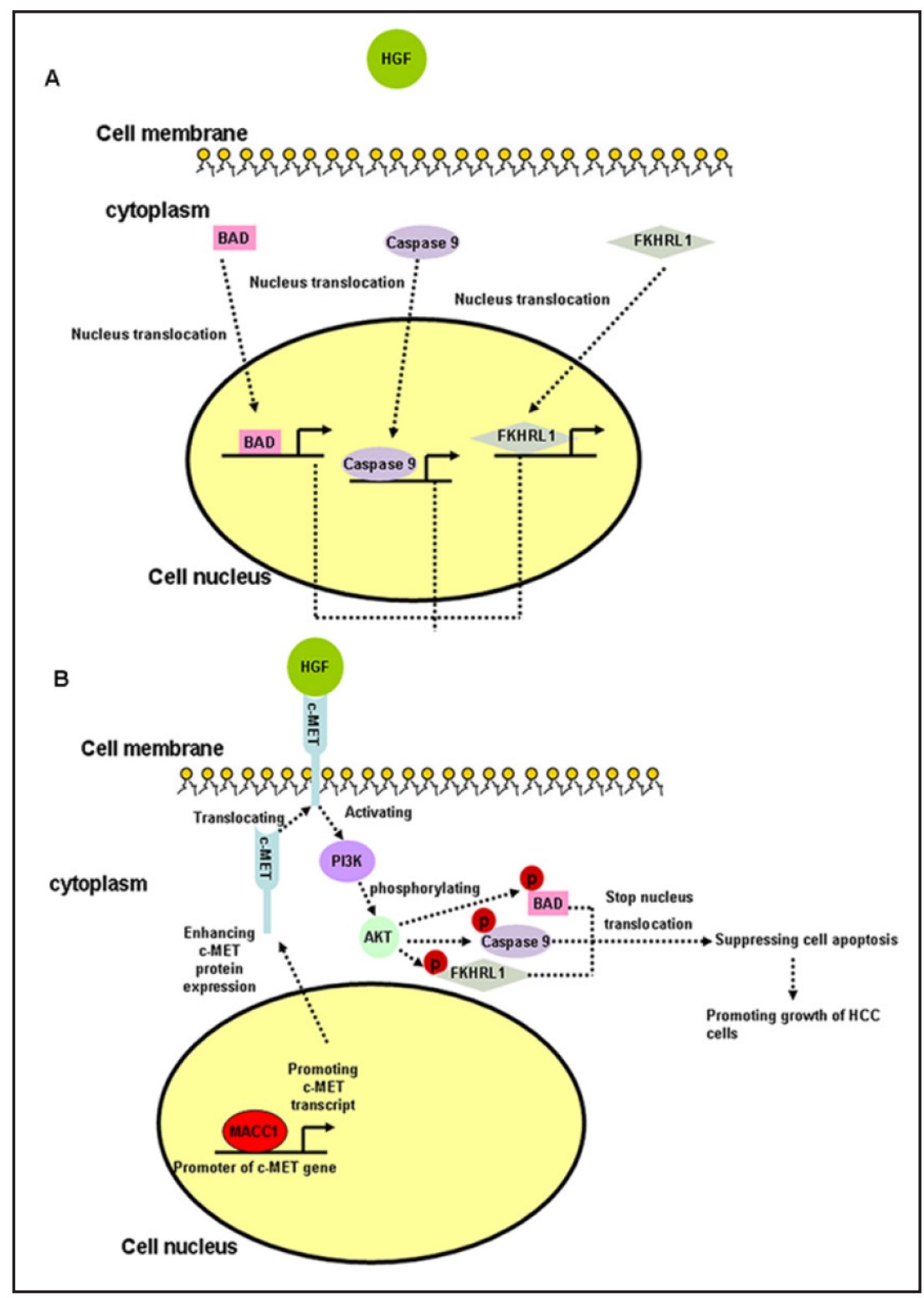

Thus, it is urgent to find a novel biomarker that not only predicts the risk of HCC recurrence after curative liver resection effectively but is also a potential therapeutic target capable of preventing HCC recurrence and treating HCC patients at advanced stages of the disease.

In this study, we reported that the expression of MACC1 was significantly increased in HCC tissues compared with adjacent liver tissues and that its expression in HCC tissues was positively associated with high Edmondson-Steiner classification and advanced TNM staging. Moreover, comparison of Kaplan-Meier survival curves indicated that patients with higher MACC1 expression in HCC tissues had notably worse prognosis after liver resection. These clinical data suggest strongly that MACC1 is closely involved in the mechanism of HCC recurrence after liver resection and functions as a potentially efficacious biomarker and therapeutic target of postsurgical HCC recurrence. These findings are consistent with the results of other groups $[16,17]$.

Mostinvestigations of the role of MACC1 in cancer progression focused on the mechanism whereby MACC1 promotes metastasis in a variety of cancers [16-22]. The present study attempted for the first time to explore its role in mediating cell apoptosis. We found that the overexpression of MACC1 promoted cell growth in both Hep3B and SMMC7721 cells 
through the repression of cell apoptosis, whereas the deletion of MACC1 led to the opposite results. In addition, we verified that the overexpression of MACC1 promoted tumor growth in a nude mouse model by repressing cell apoptosis. These results of both in vitro and in vivo experiments revealed that MACC1 had an anti-apoptotic function in HCC cells.

To discover the underlying molecular mechanism of MACC1, we examined the influence of MACC1 on HGF/c-MET signaling in HCC. Stein's group first showed in colon cancer that MACC1 regulates HGF/c-MET signaling and consequently contributes to cancer metastasis [3]. They also showed by both reporter chloramphenicol acetyltransferase and electrophoretic mobility shift assay that MACC1 protein binds the promoter of c-MET (fragment -223 to -68 base pairs upstream of its transcription start site) directly in colon cancer. We obtained similar results for HCC here, showing that forced expression of MACC1 promoted c-MET expression and c-MET phosphorylation induced by HGF in Hep3B cells. The ablation of c-MET was found to abolish the anti-apoptotic effect of MACC1 in Hep3B cells. These data demonstrate that MACC1 represses cell apoptosis in HCC by regulating c-MET directly.

Furthermore, we found that MACC1 increased HGF-driven AKT phosphorylation. The PI3K inhibitor LY294002 suppressed the phosphorylation of AKT induced by MACC1, which reveals that MACC1 mediates AKT phosphorylation by facilitating the HGF/c-MET/ PI3K pathway. BAD, Caspase 9 and FKHRL1 have already been validated in several studies to be phosphorylated by p-AKT and in turn lose their pro-apoptotic effect. Here, we found that MACC1 overexpression promoted the phosphorylation of BAD, Caspase 9 and FKHRL1 induced by HGF in Hep3B cells. These data indicate strongly that MACC1 promotes the phosphorylation of BAD, Caspase 9 and FKHRL1 through the regulation of the HGF/c-MET/ PI3K/AKT pathway and consequently inhibits cell apoptosis in HCC.

In summary, in this study we found that MACC1 overexpression predicted worse prognosis after liver resection, which was attributed to its anti-apoptotic effect on HCC cells. Furthermore, we demonstrated that its underlying mechanism was that MACC1 accelerated the activation of the HGF/c-MET/PI3K/AKT pathway, phosphorylated anti-apoptotic proteins including BAD, Caspase 9 and FKHRL1, and ultimately exerted an anti-apoptotic function (Fig. 9). Hence, MACC1 could potentially serve as a clinical biomarker and therapeutic target for HCC recurrence after liver resection.

\section{Abbreviations}

MACC1 (metastasis associated with colon cancer 1); HCC (hepatocellular carcinoma); PI3K (phosphoinositide 3-kinase); BAD (Bcl-2-associated death promoter); MAPK (Rasmitogen-activated protein kinase); PIP3 (phosphatidylinositol (3-5)-trisphosphate); PIP2

(phosphatidylinositol 4,5-bisphosphate); AFP (serum $\alpha$-fetoprotein); TUNEL (Terminal deoxynucleotidyl transferase dUTP nick end labeling); IHC (immunohistochemistry); MTT (3-(4,5-Dimethylthiazol-2-yl)-2,5-Diphenyltetrazolium Bromide).

\section{Acknowledgments}

This study was supported by grants from the National Natural Scientific Foundation of China (No. 81272645 and 81072052 to Qingguang Liu, 81071897 to Yingmin Yao and 81301743 to Xin Zheng), the Research Fund for the Doctoral Program of High Education of China from the Ministry of Education (No. 20120201120090 to Xin Zheng) and the Fundamental Research Funds for the Basic Research Operating Expenses Program of Central College sponsored by Xi'an Jiaotong University to Xin Zheng.

\section{Disclosure Statement}

No. 
Yao et al.: MACC1 Represses HCC Cell Apoptosis

\section{References}

1 Bosch FX, Ribes J, Diaz M, Cleries R: Primary liver cancer: Worldwide incidence and trends. Gastroenterology 2004;127:S5-S16.

-2 Jemal A, Bray F, Center MM, Ferlay J, Ward E, Forman D: Global cancer statistics. CA Cancer J Clin 2011;61:69-90.

3 Stein U, Walther W, Arlt F, Schwabe H, Smith J, Fichtner I, Birchmeier W, Schlag PM: Macc1, a newly identified key regulator of hgf-met signaling, predicts colon cancer metastasis. Nature Medicine 2009;15:59-67.

-4 Forte G, Minieri M, Cossa P, Antenucci D, Sala M, Gnocchi V, Fiaccavento R, Carotenuto F, De Vito P, Baldini PM, Prat M, Di Nardo P: Hepatocyte growth factor effects on mesenchymal stem cells: Proliferation, migration, and differentiation. Stem Cells 2006;24:23-33.

5 Ma PC, Maulik G, Christensen J, Salgia R: C-met: Structure, functions and potential for therapeutic inhibition. Cancer \& Metastasis Reviews 2003;22:309-325.

6 Whittaker S, Marais R, Zhu AX: The role of signaling pathways in the development and treatment of hepatocellular carcinoma. Oncogene 2010;29:4989-5005.

7 Graziani A, Gramaglia D, Cantley LC, Comoglio PM: The tyrosine-phosphorylated hepatocyte growth factor/ scatter factor receptor associates with phosphatidylinositol 3-kinase. J Biol Chem 1991;266:22087-22090.

$>8$ Ponzetto C, Bardelli A, Zhen Z, Maina F, dalla Zonca P, Giordano S, Graziani A, Panayotou G, Comoglio PM: A multifunctional docking site mediates signaling and transformation by the hepatocyte growth factor/ scatter factor receptor family. Cell 1994;77:261-271.

-9 Sakamaki J, Daitoku H, Ueno K, Hagiwara A, Yamagata K, Fukamizu A: Arginine methylation of bcl-2 antagonist of cell death (bad) counteracts its phosphorylation and inactivation by akt. Proc Natl Acad Sci U S A 2011;108:6085-6090.

10 Uddin S, Kottegoda S, Stigger D, Platanias LC, Wickrema A: Activation of the akt/fkhrl1 pathway mediates the antiapoptotic effects of erythropoietin in primary human erythroid progenitors. Biochem Biophys Res Commun 2000;275:16-19.

11 Gao J, Ding F, Liu Q Yao Y: Knockdown of macc1 expression suppressed hepatocellular carcinoma cell migration and invasion and inhibited expression of mmp2 and mmp9. Mol Cell Biochem 2013;376:21-32.

12 Zheng X, Vittar NB, Gai X, Fernandez-Barrena MG, Moser CD, Hu C, Almada LL, McCleary-Wheeler AL, Elsawa SF, Vrabel AM, Shire AM, Comba A, Thorgeirsson SS, Kim Y, Liu Q, Fernandez-Zapico ME, Roberts LR: The transcription factor gli1 mediates tgfbeta1 driven emt in hepatocellular carcinoma via a snai1dependent mechanism. PLoS One 2012;7:e49581.

13 Zheng X, Gai X, Ding F, Lu Z, Tu K, Yao Y, Liu Q: Histone acetyltransferase pcaf up-regulated cell apoptosis in hepatocellular carcinoma via acetylating histone h4 and inactivating akt signaling. Mol Cancer 2013;12:96.

14 Siegel R, Naishadham D, Jemal A: Cancer statistics, 2012. CA Cancer J Clin 2012;62:10-29.

-15 Gluer AM, Cocco N, Laurence JM, Johnston ES, Hollands MJ, Pleass HC, Richardson AJ, Lam VW: Systematic review of actual 10-year survival following resection for hepatocellular carcinoma. HPB (Oxford) 2012;14:285-290.

16 Qiu J, Huang P, Liu Q Hong J, Li B, Lu C, Wang L, Wang J, Yuan Y: Identification of macc1 as a novel prognostic marker in hepatocellular carcinoma. Journal of Translational Medicine 2011;9:166.

17 Xie C, Wu J, Yun J, Lai J, Yuan Y, Gao Z, Li M, Li J, Song L: Macc1 as a prognostic biomarker for early-stage and afp-normal hepatocellular carcinoma. PLoS One 2013;8:e64235.

18 Shirahata A, Shinmura K, Kitamura Y, Sakuraba K, Yokomizo K, Goto T, Mizukami H, Saito M, Ishibashi K, Kigawa G, Nemoto H, Hibi K: Macc1 as a marker for advanced colorectal carcinoma. Anticancer research 2010;30:2689-2692.

-19 Stein U, Burock S, Herrmann P, Wendler I, Niederstrasser M, Wernecke KD, Schlag PM: Circulating macc1 transcripts in colorectal cancer patient plasma predict metastasis and prognosis. PLoS One 2012;7:e49249.

20 Wang G, Kang MX, Lu WJ, Chen Y, Zhang B, Wu YL: Macc1: A potential molecule associated with pancreatic cancer metastasis and chemoresistance. Oncology Letters 2012;4:783-791.

21 Wang L, Wu Y, Lin L, Liu P, Huang H, Liao W, Zheng D, Zuo Q Sun L, Huang N, Shi M, Liao Y: Metastasisassociated in colon cancer-1 upregulation predicts a poor prognosis of gastric cancer, and promotes tumor cell proliferation and invasion. Int J Cancer 2013;133:1419-1430.

22 Zhang Y, Wang Z, Chen M, Peng L, Wang X, Ma Q, Ma F, Jiang B: Microrna-143 targets macc1 to inhibit cell invasion and migration in colorectal cancer. Molecular Cancer 2012;11:23. 